\title{
Prefix exchanging and pattern avoidance by involutions
}

\author{
Aaron D. Jaggard* \\ Department of Mathematics \\ Tulane University \\ New Orleans, LA 70118 USA \\ adj@math.tulane.edu
}

Submitted: May 26, 2003; Accepted: Sep 16, 2003; Published: Sep 22, 2003

MR Subject Classifications: 05A05, 05A15

\begin{abstract}
Let $I_{n}(\pi)$ denote the number of involutions in the symmetric group $\mathcal{S}_{n}$ which avoid the permutation $\pi$. We say that two permutations $\alpha, \beta \in \mathcal{S}_{j}$ may be exchanged if for every $n, k$, and ordering $\tau$ of $j+1, \ldots, k$, we have $I_{n}(\alpha \tau)=I_{n}(\beta \tau)$. Here we prove that 12 and 21 may be exchanged and that 123 and 321 may be exchanged. The ability to exchange 123 and 321 implies a conjecture of Guibert, thus completing the classification of $\mathcal{S}_{4}$ with respect to pattern avoidance by involutions; both of these results also have consequences for longer patterns.

Pattern avoidance by involutions may be generalized to rook placements on Ferrers boards which satisfy certain symmetry conditions. Here we provide sufficient conditions for the corresponding generalization of the ability to exchange two prefixes and show that these conditions are satisfied by 12 and 21 and by 123 and 321 . Our results and approach parallel work by Babson and West on analogous problems for pattern avoidance by general (not necessarily involutive) permutations, with some modifications required by the symmetry of the current problem.
\end{abstract}

\section{Introduction}

The pattern of a sequence $w=w_{1} w_{2} \ldots w_{k}$ of $k$ distinct letters is the order-preserving relabelling of $w$ with $[k]=\{1,2, \ldots, k\}$. Given a permutation $\pi=\pi_{1} \pi_{2} \ldots \pi_{n}$ in the

*This work is drawn from the author's Ph.D. dissertation which was written at the University of Pennsylvania under the supervision of Herbert S. Wilf. The author was partially supported by the DoD University Research Initiative (URI) program administered by the ONR under grant N00014-01-1-0795; the presentation of this work at the Permutation Patterns 2003 conference was partially supported by Penn GAPSA and the New Zealand Institute for Mathematics and its Applications. 
symmetric group $\mathcal{S}_{n}$, we say that $\pi$ avoids the pattern $\sigma=\sigma_{1} \sigma_{2} \ldots \sigma_{k} \in \mathcal{S}_{k}$ if there is no subsequence $\pi_{i_{1}} \ldots \pi_{i_{k}}, i_{1}<\cdots<i_{k}$, whose pattern is $\sigma$.

Let $\mathcal{I}_{n}(\sigma)$ denote the number of involutions in $\mathcal{S}_{n}$ (permutations whose square is the identity permutation) which avoid the pattern $\sigma$, and write $\sigma \sim_{\mathcal{I}} \sigma^{\prime}$ if for every $n, \mathcal{I}_{n}(\sigma)=$ $\mathcal{I}_{n}\left(\sigma^{\prime}\right)$. (In this case we also say that $\sigma$ and $\sigma^{\prime}$ are $\sim_{\mathcal{I}}$-equivalent.) For $\alpha, \beta \in \mathcal{S}_{j}$, we say that the prefixes $\alpha$ and $\beta$ may be exchanged if for every $k \geq j$ and ordering $\tau=\tau_{j+1} \tau_{j+2} \ldots \tau_{k}$ of $[k] \backslash[j]$, the patterns $\alpha_{1} \ldots \alpha_{j} \tau_{j+1} \tau_{j+2} \ldots \tau_{k}$ and $\beta_{1} \ldots \beta_{j} \tau_{j+1} \tau_{j+2} \ldots \tau_{k}$ are $\sim_{\mathcal{I}^{-} \text {equivalent. }}$

Our work here implies the following corollaries about the ability to exchange certain prefixes. These results and the techniques we use throughout this paper closely parallel work by Babson and West [BW00] on pattern avoidance by general permutations (without the restriction to involutions).

Corollary 4.3. The prefixes 12 and 21 may be exchanged.

Corollary 5.4. The prefixes 123 and 321 may be exchanged.

Corollary 5.4 implies an affirmative answer to a conjecture of Guibert (that $1234 \sim_{\mathcal{I}} 1432$ ) and thus completes the classification of $\mathcal{S}_{4}$ according to $\sim_{\mathcal{I}^{-}}$equivalence. Corollaries 4.3 and 5.4 also imply $\sim_{\mathcal{I}^{-}}$equivalences for patterns of length greater than 4 ; we discuss these in some detail for patterns in $\mathcal{S}_{5}$.

These corollaries follow from the sufficient conditions for exchanging prefixes given by Corollary 3.7. Recent work by Stankova and West [SW02] and Reifegerste [Rei03] on different aspects of pattern avoidance by general permutations suggests the generalization of Corollary 3.7 given by Theorem 3.1 below. In order to state this theorem, we need the following definitions.

Definition 1.1. Given a (Ferrers) shape $\lambda$, a placement on $\lambda$ is an assignment of dots to some of the boxes in $\lambda$ such that no row or column contains more than one dot. We call a placement on $\lambda$ full if each row and column of $\lambda$ contains exactly 1 dot. We define the transpose of a placement to be the placement which has a dot in box $(i, j)$ if and only if the original placement had a dot in box $(j, i)$. We call a placement on a shape $\lambda$ symmetric if the transpose of the placement is the original placement.

The transpose of a placement on a shape $\lambda$ is a placement on the conjugate shape $\lambda^{\prime}$ of $\lambda$. We use 'self-conjugate' to describe the symmetry of shapes and 'symmetric' to describe the symmetry of placements on shapes; our work makes use of symmetric placements on self-conjugate shapes.

Figure 1 shows four placements on the self-conjugate shape $\lambda=(3,3,2)$. The placement on the far left has one dot and is not full. The placement on the center left of the figure is full but not symmetric; its transpose is shown at the center right of this figure. Finally, the placement on the far right is a symmetric full placement, with the dashed line indicating the symmetry of the placement.

Pattern containment can be generalized to placements on shapes (as in, e.g., [BW00]) as follows. 

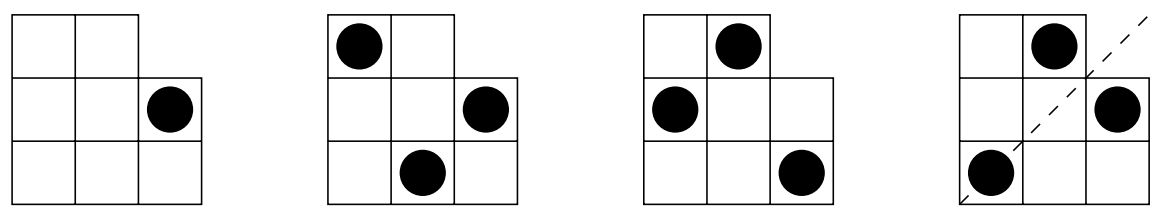

Figure 1: Four placements on the self-conjugate shape $(3,3,2)$.

Definition 1.2. A placement on a shape $\lambda$ contains the pattern $\sigma$ if there is a set $\left\{\left(x_{i}, y_{i}\right)\right\}_{i \in[j]}$ of $j$ dots in the placement which are in the same relationship as the values of $\sigma$ (i.e., $x_{1}<\cdots<x_{j}$ and the pattern of $y_{1} \ldots y_{j}$ is $\sigma$ ) and which are bounded by a rectangular subshape of $\lambda$.

Example 1.3. Figure 2 shows a placement on the shape $(3,3,2)$ which contains the patterns 12 and 21; dots whose heights form these patterns are bounded by the shaded rectangular subshapes of $(3,3,2)$ indicated in the center left and right of Figure 2. This placement does not contain the pattern 231 because, although the heights of the dots in the placement form the pattern 231, the smallest rectangular shape (shaded, far right) which bounds all three of these dots is not a subshape of $(3,3,2)$.
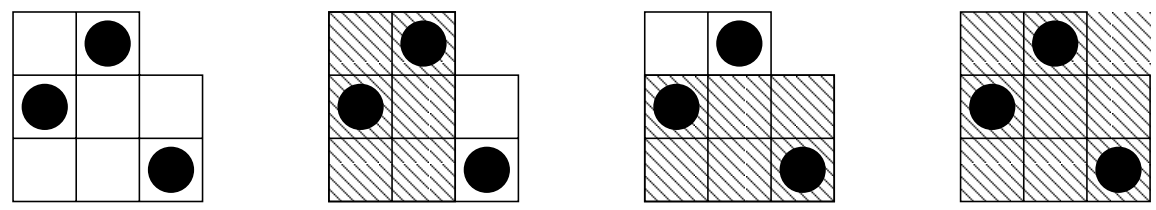

Figure 2: A placement on $(3,3,2)$ which contains the patterns 12 and 21 but not the pattern 231

With these definitions in hand, we may state the most general theorem that we prove here.

Theorem 3.1. Let $\lambda_{\text {sym }}(T)$ be the number of symmetric full placements on the shape $\lambda$ which avoid all of the patterns in the set T. Let $\alpha$ and $\beta$ be involutions in $\mathcal{S}_{j}$. Let $T_{\alpha}$ be a set of patterns, each of which begins with the prefix $\alpha$, and $T_{\beta}$ be the set of patterns obtained by replacing in each pattern in $T_{\alpha}$ the prefix $\alpha$ with the prefix $\beta$. If for every self-conjugate shape $\lambda \lambda_{\text {sym }}(\{\alpha\})=\lambda_{\text {sym }}(\{\beta\})$, then for every self-conjugate shape $\mu$

$$
\mu_{\text {sym }}\left(T_{\alpha}\right)=\mu_{\text {sym }}\left(T_{\beta}\right) .
$$

Here we also prove that the conditions on $\alpha$ and $\beta$ in Theorem 3.1 are satisfied by the patterns 12 and 21 (Theorem 4.2) and by 123 and 321 (Theorem 5.3). Corollaries 4.3 and 5.4 then follow.

Section 2 reviews the work mentioned above and other relevant literature and gives some additional basic definitions. Section 3 contains some general theorems related to 
involutions and patterns. In Sections 4 and 5 we show that we can apply this general machinery to the prefixes 12 and 21 and then to 123 and 321. Finally, in Section 6 we discuss some $\sim_{\mathcal{I}^{-}}$equivalences implied by our work as well as some interesting open questions.

\section{Background}

\subsection{General preliminaries}

We make use of the following representation of a permutation.

Definition 2.1. The graph of a permutation $\pi \in \mathcal{S}_{n}$ is an $n \times n$ array of boxes with dots in exactly the set of boxes $\{(i, \pi(i))\}_{i \in[n]}$.

The graph of $\pi^{-1}$ has a dot in the box $(x, y)$ if and only if the graph of $\pi$ has a dot in the box $(y, x)$. We coordinatize the graphs of permutations from the bottom left corner, so a permutation is an involution if and only if its graph is symmetric about the diagonal connecting its bottom left and top right corners. The graphs of 497385621 (a non-involution) and 127965384 (an involution) are shown in Figure 3, with the dashed line indicating the symmetry which characterizes graphs of involutions. Denoting by $S Q_{n}$ the $n \times n$ square shape, the graphs of involutions in $\mathcal{S}_{n}$ are exactly the symmetric full placements on $S Q_{n}$.
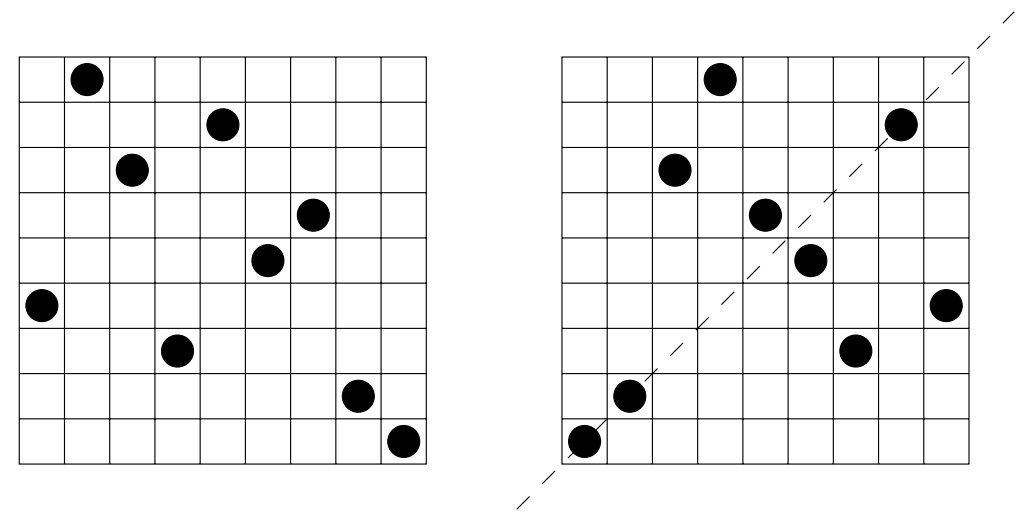

Figure 3: The graphs of the non-involution 497385621 (left) and the involution 127965384 (right).

We will make use of the Robinson-Schensted-Knuth (RSK) algorithm, which is treated in both Chapter 7 and Appendix 1 of [Sta99]. The RSK algorithm gives a bijection between permutations in $\mathcal{S}_{n}$ and pairs $(P, Q)$ of standard Young tableaux such that the shape of $P$ is that of $Q$ and this common shape has $n$ boxes. If $\pi \leftrightarrow(P, Q)$, then $\pi^{-1} \leftrightarrow(Q, P)$, so this gives a bijection between $n$-involutions and single tableaux of size $n$. 
The Schützenberger involution, or evacuation, is an operation on tableaux. Given a tableau $Q$, it produces a tableau $\operatorname{evac}(Q)$ of the same shape as $Q$ and such that $\operatorname{evac}(\operatorname{evac}(Q))=Q$. A complete development of this operation is given in Appendix 1 of [Sta99]. We note here the following property, due to Schützenberger, which is given as Corollary A1.2.11 in [Sta99].

Proposition 2.2 (Schützenberger [Sch63]). Let $w=w_{1} \ldots w_{n} \leftrightarrow(P, Q)$. Then

$$
w_{n} \ldots w_{1} \leftrightarrow\left(P^{t}, \operatorname{evac}(Q)^{t}\right)
$$

where $P^{t}$ denotes the transpose of the tableau $P$.

\subsection{Pattern avoidance background}

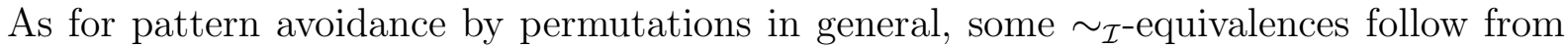
symmetry considerations. Four of the symmetries of the square preserve the symmetry which characterizes the graphs of involutions. The images of a pattern $\tau$ under these symmetries are patterns which are trivially $\sim_{\mathcal{I}^{-}}$equivalent to $\tau$; these patterns form the (involution) symmetry class of $\tau$. For $\tau \in \mathcal{S}_{k}$ these patterns are $\tau, \tau^{-1}$, the reversed complement $\tau_{r c}=\left(k+1-\tau_{k}\right) \ldots\left(k+1-\tau_{2}\right)\left(k+1-\tau_{1}\right)$ of $\tau$, and $\left(\tau_{r c}\right)^{-1}$. Since we cannot use all 8 of the symmetries of the square, each symmetry class which arises in pattern avoidance by general permutations may split into 2 involution symmetry classes.

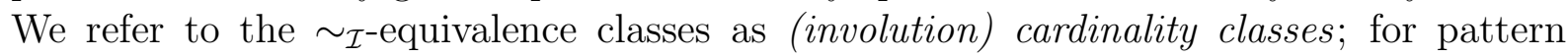
avoidance by general permutations, the cardinality classes are usually referred to as Wilf classes. Unless otherwise stated, we take 'symmetry' and 'cardinality' classes to be with respect to $\sim_{\mathcal{I}}$, and use ' $\sim_{\mathcal{S}^{-}}$' to indicate equivalence with respect to pattern avoidance by general permutations.

In their well-known paper [SS85], Simion and Schmidt found the cardinality classes of $\mathcal{S}_{3}$ and proved the following propositions.

Proposition 2.3 (Simion and Schmidt [SS85]). For $\tau \in\{123,132,213,321\}$ and $n \geq 1$,

$$
\mathcal{I}_{n}(\tau)=\left(\begin{array}{c}
n \\
{[n / 2]}
\end{array}\right)
$$

Proposition 2.4 (Simion and Schmidt [SS85]). For $\tau \in\{231,312\}$ and $n \geq 1$,

$$
\mathcal{I}_{n}(\tau)=2^{n-1} \text {. }
$$

Comparing this to the classic result that $\mathcal{S}_{3}$ contains a single Wilf class, we see that passing from symmetry to cardinality classes does not repair all of the breaks in $\sim_{\mathcal{S}}$-symmetry classes caused by considering pattern avoidance by involutions instead of general permutations.

Many of the sequences $\left\{\mathcal{I}_{n}(\tau)\right\}$ which are known are for $\tau=12 \ldots k$, in which case the sequence counts the number of standard tableaux of size $n$ with at most $k-1$ columns. A theorem of Regev covers $k=4$ as follows. 
Proposition 2.5 (Regev [Reg81]).

$$
\mathcal{I}_{n}(1234)=\sum_{i=0}^{\lfloor n / 2\rfloor}\left(\begin{array}{c}
n \\
2 i
\end{array}\right)\left(\begin{array}{c}
2 i \\
i
\end{array}\right) \frac{1}{i+1},
$$

i.e., the $n^{\text {th }}$ Motzkin number $M_{n}$.

Regev also gave the following expression for the asymptotic value of $\mathcal{I}_{n}(12 \ldots k)$.

Theorem 2.6 (Regev [Reg81]).

$$
\mathcal{I}_{n}(12 \ldots k(k+1)) \sim k^{n}\left(\frac{k}{n}\right)^{k(k-1) / 4} \frac{1}{k !} \Gamma\left(\frac{3}{2}\right)^{-k} \prod_{j=1}^{k} \Gamma\left(1+\frac{j}{2}\right)
$$

Gouyou-Beauchamps studied Young tableaux of bounded height in [GB89] and obtained exact results for $k=5$ and 6 .

Proposition 2.7 (Gouyou-Beauchamps [GB89]).

$$
\mathcal{I}_{n}(12345)=\left\{\begin{array}{ll}
C_{k} C_{k}, & n=2 k-1 \\
C_{k} C_{k+1}, & n=2 k
\end{array},\right.
$$

where $C_{k}=\frac{1}{k+1}\left(\begin{array}{c}2 k \\ k\end{array}\right)$, the $k^{\text {th }}$ Catalan number.

Proposition 2.8 (Gouyou-Beauchamps [GB89]).

$$
\mathcal{I}_{n}(123456)=\sum_{i=0}^{\lfloor n / 2\rfloor} \frac{3 ! n !(2 i+2) !}{(n-2 i) ! i !(i+1) !(i+2) !(i+3) !} .
$$

Gessel [Ges90] has given a determinantal formula for the general $\mathcal{I}_{n}(12 \ldots k)$.

Work of Guibert and others has almost completely determined the cardinality classes of $\mathcal{S}_{4}$ (see [GPP01] for a review of this work). Symmetry of the RSK algorithm implies $1234 \sim_{\mathcal{I}} 4321$. Guibert bijectively obtained the following results in his thesis [Gui95].

Proposition 2.9 (Guibert [Gui95]).

$$
3412 \sim_{\mathcal{I}} 4321
$$

Proposition 2.10 (Guibert [Gui95]).

$$
2143 \sim_{\mathcal{I}} 1243
$$

Guibert also conjectured that both $\mathcal{I}_{n}(2143)$ and $\mathcal{I}_{n}(1432)$ are equal to $M_{n}$ for $n \geq 4$ (as $\mathcal{I}_{n}(1234)$ is known to be). Guibert, Pergola, and Pinzani [GPP01] affirmatively answered the first of these conjectures. 


\section{Proposition 2.11 (Guibert, Pergola, Pinzani [GPP01]).}

$$
1234 \sim_{\mathcal{I}} 2143
$$

In more recent work on involutions avoiding various combinations of multiple patterns, Guibert and Mansour [GM02] noted that the second conjecture was still open. We prove that conjecture as Corollary 6.2.

There are various known $\sim_{\mathcal{S}}$-equivalences between $\sim_{\mathcal{S}}$-symmetry classes. Of particular interest are those which follow from more general theorems, which we review here. In Sections 3-5 we prove the first such general theorems for pattern avoidance by involutions.

West proved the following theorem in his thesis [Wes90].

Theorem 2.12 (West [Wes90]). For any $k$, any ordering $\tau=\tau_{3} \ldots \tau_{k}$ of $[k] \backslash[2]$, and any $n$, the number of permutations in $\mathcal{S}_{n}$ which avoid the pattern $12 \tau_{3} \ldots \tau_{k}$ equals the number of permutations in $\mathcal{S}_{n}$ which avoid $21 \tau_{3} \ldots \tau_{k}$.

Babson and West [BW00] restated the proof of Theorem 2.12 and then proved the following theorem.

Theorem 2.13 (Babson and West [BW00]). For any $k$, any ordering $\tau=\tau_{4} \ldots \tau_{k}$ of $[k] \backslash[3]$, and any $n$, the number of permutations in $\mathcal{S}_{n}$ which avoid the pattern $123 \tau_{4} \ldots \tau_{k}$ equals the number of permutations in $\mathcal{S}_{n}$ which avoid $321 \tau_{4} \ldots \tau_{k}$.

Stankova and West [SW02] further investigated the property, which they called shapeWilf-equivalence, used by Babson and West in their proofs of these two theorems. Two patterns $\alpha$ and $\beta$ are shape-Wilf-equivalent if, for every shape $\lambda$, the number of full placements on $\lambda$ which avoid $\alpha$ equals the number which avoid $\beta$; this implies the Wilfequivalence of the patterns in question. Stankova and West proved that the patterns $231 \tau_{4} \ldots \tau_{k}$ and $312 \tau_{4} \ldots \tau_{k}$ are shape-Wilf-equivalent. More recently, Backelin, West, and Xin [BWX] have proved that the patterns $12 \ldots j \tau_{j+1} \ldots \tau_{k}$ and $j \ldots 21 \tau_{j+1} \ldots \tau_{k}$ are shape-Wilf-equivalent. Here we define and use a symmetrized version of shape-Wilfequivalence.

Finally, a recent paper by Reifegerste [Rei03] generalizes a bijection given by Simion and Schmidt. One application (Corollary 9 of [Rei03]) is that a certain set of patterns with prefix 12 is as restrictive (with respect to pattern avoidance by general permutations) as the set of patterns obtained by replacing these occurrences of 12 with 21; this suggests part of our most general result below.

\section{Some General Machinery}

In order to prove Corollaries 4.3 and 5.4 we need only Corollary 3.7 below and some additional lemmas. The recent work, discussed in Section 2, by Reifegerste and by Stankova and West suggests the generalization of Corollary 3.7 given by Theorem 3.1. 
Theorem 3.1. Let $\lambda_{\text {sym }}(T)$ be the number of symmetric full placements on the shape $\lambda$ which avoid all of the patterns in the set $T$. Let $\alpha$ and $\beta$ be involutions in $\mathcal{S}_{j}$. Let $T_{\alpha}$ be a set of patterns, each of which begins with the prefix $\alpha$, and $T_{\beta}$ be the set of patterns obtained by replacing in each pattern in $T_{\alpha}$ the prefix $\alpha$ with the prefix $\beta$. If for every self-conjugate shape $\lambda \lambda_{\text {sym }}(\{\alpha\})=\lambda_{\text {sym }}(\{\beta\})$, then for every self-conjugate shape $\mu$

$$
\mu_{\text {sym }}\left(T_{\alpha}\right)=\mu_{\text {sym }}\left(T_{\beta}\right) .
$$

The proof of Theorem 3.1 makes use of the following definition.

Definition 3.2. Fix positive integers $j$ and $l$, and for every $i \in[l]$ let $\tau_{i}$ be an ordering of $\left[k_{i}\right] \backslash[j]$ for some $k_{i} \geq j$. Let $T$ be the set $\left\{\tau_{i}\right\}_{i \in[l]}$ and $\mu$ be a self-conjugate shape with a symmetric full placement $P$. We construct the self-conjugate T-shape of $(\mu, P)$, denoted $\lambda_{T}(\mu, P)$, as follows; Example 3.3 and Figure 4 below illustrate this procedure.

Take all boxes $(x, y)$ and $(y, x)$ in $\mu$ such that $(x, y)$ is strictly southwest of an occurrence of the pattern of some $\tau_{i} \in T$ (i.e., for which there is a set of $k_{i}-j$ dots, contained within a rectangular subshape of $\mu$, whose heights have pattern $\tau_{i}$ and which are all above and to the right of $(x, y)$.) This set of boxes forms a self-conjugate shape, since it contains $(x, y)$ iff it contains $(y, x)$, on which there is a (not necessarily full) symmetric placement obtained by restricting $P$ to this shape. Delete the rows and columns of this shape which do not contain a dot to obtain the self-conjugate shape $\lambda_{T}(\mu, P)$. The deletion of empty rows and columns yields a symmetric full placement on $\lambda_{T}(\mu, P)$; we call this the placement on $\lambda_{T}(\mu, P)$ induced by $P$.

Example 3.3. We view the graph of 127965384, shown in the right part of Figure 3, as a placement $P$ on $\mu=S Q_{9}$ and let $T=\{54\}$. The left of Figure 4 shows this graph with shading added to those boxes which are southwest of some pair of dots whose pattern is 21 (the pattern of $54 \in T$ ) or which are the reflection of such a box across the diagonal of symmetry. Removing the empty rows and columns from this shaded shape, we obtain $\lambda_{\{54\}}\left(S Q_{9}, P\right)$ and the placement on $\lambda_{\{54\}}\left(S Q_{9}, P\right)$ induced by $P$; these are shown at the far right. The center right of Figure 4 shows the graph of 127965384 with the boxes corresponding to $\lambda_{\{54\}}\left(S Q_{9}, P\right)$ crossed out.

Remark 3.4. The shape of $\lambda_{T}(\mu, P)$ does not depend on the placement induced on it by $P$. For any placement $P^{\prime}$ on $\mu$ which agrees with $P$ outside of the boxes corresponding to $\lambda_{T}(\mu, P)$, we have $\lambda_{T}\left(\mu, P^{\prime}\right)=\lambda_{T}(\mu, P)$.

The motivation for the definition of $\lambda_{T}(\mu, P)$ is that it satisfies the following lemma.

Lemma 3.5. Fix positive integers $j$ and $l$, and for every $i \in[l]$ let $\tau_{i}$ be an ordering of

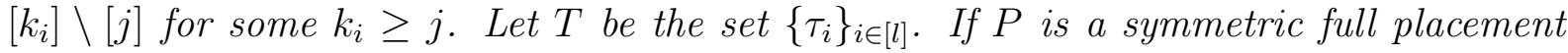
on a self-conjugate shape $\mu$ and $\sigma$ is a $j$-involution, then $P$ contains at least one of the patterns $\left\{\sigma \tau_{i}\right\}_{i \in[l]}$ if and only if the placement on $\lambda_{T}(\mu, P)$ induced by $P$ contains $\sigma$. 


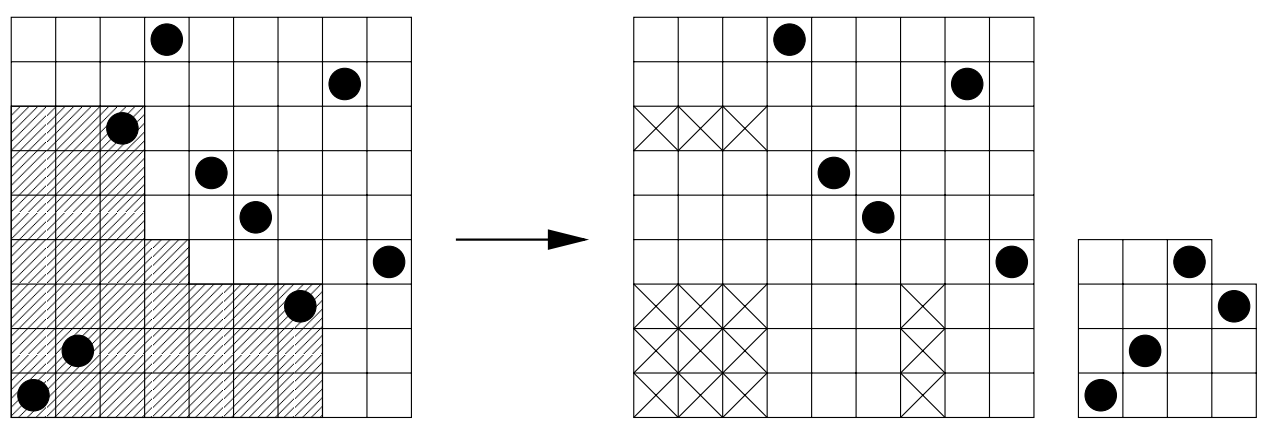

Figure 4: Constructing $\lambda_{T}(\mu, P)$.

Proof. Assume $P$ contains an occurrence of $\sigma \tau_{i}$ in the boxes $\left(x_{1}, y_{1}\right), \ldots,\left(x_{k_{i}}, y_{k_{i}}\right)$. The box $\left(x_{j}, \max _{1 \leq m \leq j}\left\{y_{m}\right\}\right)$ is southwest of all of the dots in the boxes $\left(x_{j+1}, y_{j+1}\right), \ldots$, $\left(x_{j+k_{i}}, y_{j+k_{i}}\right)$, whose pattern is that of $\tau_{i}$. The boxes $\left(x_{1}, y_{1}\right), \ldots,\left(x_{j}, y_{j}\right)$, whose pattern is $\sigma$, are all (weakly) southwest of this box and are thus contained in a rectangular subshape of $\lambda_{T}(\mu, P)$. The placement on $\lambda_{T}(\mu, P)$ induced by $P$ thus contains the pattern $\sigma$.

If the placement on $\lambda_{T}(\mu, P)$ induced by $P$ contains $\sigma$, we consider the top right corner $(x, y)$ of a rectangular subshape of $\lambda_{T}(\mu, P)$ that bounds a set of dots which form an occurrence of $\sigma$. This corresponds (after replacing the rows and columns deleted in the construction of $\left.\lambda_{T}(\mu, P)\right)$ to a box $\left(x^{\prime}, y^{\prime}\right)$ in $\mu$ such that either $\left(x^{\prime}, y^{\prime}\right)$ or $\left(y^{\prime}, x^{\prime}\right)$ is strictly southwest of some set of $k_{i}-j$ dots whose pattern is that of some $\tau_{i} \in T$ and which are all (weakly) southwest of some box in the shape $\mu$. If the box $\left(x^{\prime}, y^{\prime}\right)$ satisfies this condition, then the original $j$ dots whose pattern is $\sigma$ together with the $k_{i}-j$ dots just found give a $\sigma \tau_{i} \in T$ pattern contained in the placement $P$. If it does not satisfy this condition, then by construction of $\lambda_{T}(\mu, P)$ the box $\left(y^{\prime}, x^{\prime}\right)$ must do so. The reflection of the set of $j$ dots which are southwest of $\left(x^{\prime}, y^{\prime}\right)$ and whose pattern is $\sigma$ is a set of $j$ dots which are southwest of $\left(y^{\prime}, x^{\prime}\right)$ and whose pattern is $\sigma^{-1}=\sigma$. These dots combine with the $k_{i}-j$ dots strictly northeast of $\left(y^{\prime}, x^{\prime}\right)$ whose pattern is $\tau_{i}$ (and which are southwest of some box in $\mu$ ) to form the pattern $\sigma \tau_{i} \in T$ contained in the placement $P$.

Example 3.6. Applying Lemma 3.5 to the involution 127965384 from Example 3.3, we see that 127965384 contains 12354 (respectively 32154 ) iff the placement on $\left(4^{3}, 3\right)$ shown at the right of Figure 4 contains 123 (respectively 321).

We now prove Theorem 3.1.

Proof of Theorem 3.1. Let $T$ be obtained from $T_{\alpha}$ by removing the prefix $\alpha$ from every pattern in $T_{\alpha}$. (Removing $\beta$ from every pattern in $T_{\beta}$ also yields $T$.) For a symmetric full placement $P$ on $\mu$, find $\lambda_{T}(\mu, P)$ and note which boxes in $\mu$ correspond to the boxes of $\lambda_{T}(\mu, P)$. Let $[P]$ be the set of symmetric full placements on $\mu$ which agree with $P$ outside of the boxes corresponding to $\lambda_{T}(\mu, P)$. By Remark 3.4, $\lambda_{T}\left(\mu, P^{\prime}\right)=\lambda_{T}(\mu, P)$ for every placement $P^{\prime} \in[P]$ and the number of placements in $[P]$ equals the number of symmetric full placements on $\lambda_{T}(\mu, P)$. By Lemma 3.5, the number of symmetric full placements in 
$[P]$ which avoid $T_{\alpha}$ (respectively $T_{\beta}$ ) equals the number $\left(\lambda_{T}(\mu, P)\right)_{\text {sym }}(\{\alpha\})$ (respectively $\left.\left(\lambda_{T}(\mu, P)\right)_{\text {sym }}(\{\beta\})\right)$ of symmetric full placements on $\lambda_{T}(\mu, P)$ which avoid $\alpha$ (respectively $\beta)$. By hypothesis $\left(\lambda_{T}(\mu, P)\right)_{\text {sym }}(\{\alpha\})=\left(\lambda_{T}(\mu, P)\right)_{\text {sym }}(\{\beta\})$, and the theorem follows by summing over all classes $[P]$.

A special case of Theorem 3.1 gives sufficient conditions for the exchange of two prefixes $\alpha$ and $\beta$.

Corollary 3.7. Let $\lambda_{\text {sym }}(\{\sigma\})$ be the number of symmetric full placements on the shape $\lambda$ which avoid the pattern $\sigma$. Let $\alpha$ and $\beta$ be involutions in $\mathcal{S}_{j}$. If, for every self-conjugate shape $\lambda$ we have $\lambda_{\text {sym }}(\{\alpha\})=\lambda_{\text {sym }}(\{\beta\})$, then the prefixes $\alpha$ and $\beta$ may be exchanged.

Proof. For any $k$ and ordering $\tau$ of $[k] \backslash[j]$, take $T_{\alpha}=\{\alpha \tau\}, T_{\beta}=\{\beta \tau\}$, and $\mu=$ $S Q_{n}$. Theorem 3.1 then implies that $\mu_{\text {sym }}(\{\alpha \tau\})=\mu_{\text {sym }}(\{\beta \tau\})$. As the symmetric full placements on $\mu$ are exactly the graphs of $n$-involutions, we have $\mathcal{I}_{n}(\alpha \tau)=\mathcal{I}_{n}(\beta \tau)$. Since this does not depend on our choices of $n$ or $\tau$, the prefixes $\alpha$ and $\beta$ may be exchanged.

\section{The patterns 12 and 21}

We now show that the conditions on $\{\alpha, \beta\}$ in Theorem 3.1 are satisfied by the patterns 12 .

Lemma 4.1. For any self-conjugate shape $\lambda$, the number $\lambda_{\text {sym }}(\{12\})$ of symmetric full placements on $\lambda$ which avoid 12 equals the number $\lambda_{\text {sym }}(\{21\})$ which avoid 21.

Proof. Babson and West [BW00] showed that if $\lambda$ has any full placements, there is a unique full placement on $\lambda$ which avoids 12 and a unique full placement on $\lambda$ which avoids 21. If $\lambda$ is self-conjugate, the reflection of any placement on $\lambda$ across the diagonal of symmetry gives another placement on $\lambda$. This placement avoids 12 (21, respectively) iff the original placement did. By the uniqueness of the full placements which avoid 12 and 21 , the reflected placement must coincide with the original one and is thus symmetric.

We may thus apply Theorem 3.1 to 12 and 21 in order to obtain the following result.

Theorem 4.2. Let $T_{12}$ be a set of patterns, each of which begins with the prefix 12, and $T_{21}$ be the set of patterns obtained by replacing in each pattern in $T_{12}$ the prefix 12 with the prefix 21 . Let $\mu_{\text {sym }}(T)$ be the number of symmetric full placements on the shape $\mu$ which avoid every pattern in the set $T$. For every self-conjugate shape $\mu$,

$$
\mu_{\text {sym }}\left(T_{12}\right)=\mu_{\text {sym }}\left(T_{21}\right) \text {. }
$$

As a corollary (also seen by applying Corollary 3.7 to Lemma 4.1), we may exchange the prefixes 12 and 21.

Corollary 4.3. The prefixes 12 and 21 may be exchanged.

We apply Corollary 4.3 to specific patterns in Section 6. 


\section{The patterns 123 and 321}

We now turn to the prefixes 123 and 321 and show that these satisfy the conditions given in Theorem 3.1. Our approach closely parallels that used by Babson and West in their work on pattern avoiding permutations that we discussed in Section 2. We symmetrize many of their results here; as we do so, we encounter problems with symmetric full placements on square shapes (i.e., the graphs of involutions). We are able to work around these problems using various symmetry properties of the RSK algorithm.

We start with the statement of the main lemma we use to apply Theorem 3.1 to the patterns 123 and 321. It relates the number of 123- and 321-avoiding symmetric full placements on self-conjugate shapes according to the position of the dot in the top row.

Lemma 5.1. If $\lambda=\left(\lambda_{1}, \ldots, \lambda_{k}\right)$ is a non-square self-conjugate shape then, for $1 \leq i \leq \lambda_{k}$, the number of symmetric full placements on $\lambda$ which avoid 123 and have a dot in $\left(i, \lambda_{1}\right)$ equals the number of symmetric full placements on $\lambda$ which avoid 321 and have a dot in $\left(\lambda_{k}+1-i, \lambda_{1}\right)$.

We defer the proof of this lemma until the end of this section and start with an example showing that the non-square condition in Lemma 5.1 is required.

Example 5.2. The conclusion of Lemma 5.1 need not hold for square shapes. Figure 5 shows the four symmetric full placements on $S Q_{3}$. The three rightmost placements avoid 123 and have dots in $(i, 3)=(2,3),(3,3)$, and $(1,3)$. The three leftmost placements avoid 321 and have dots in $(4-i, 3)=(3,3),(2,3)$, and $(3,3)(i=1,2,1)$.

The two symmetric full placements on the non-square shape $(3,3,2)$ are shown in Figure 6; each avoids both 123 and 321. The dots appearing at $(i, 3)$ are $(2,3)$ and $(1,3)$, while the dots at $(3-i, 3)$ are $(2,3)$ and $(1,3)(i=1,2)$.
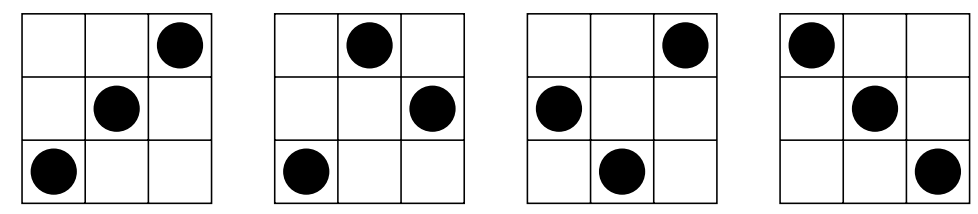

Figure 5: The conclusion of Lemma 5.1 does not hold for the square shape $S Q_{3}$.
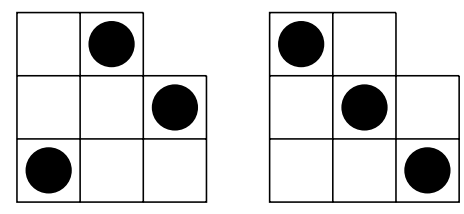

Figure 6: Illustration of Lemma 5.1 for the non-square shape $(3,3,2)$.

We now apply Theorem 3.1 to the patterns 123 and 321. 
Theorem 5.3. Let $T_{123}$ be a set of patterns, each of which begins with the prefix 123, and $T_{321}$ be the set of patterns obtained by replacing in each pattern in $T_{123}$ the prefix 123 with the prefix 321. Let $\mu_{\text {sym }}(T)$ be the number of symmetric full placements on the shape $\mu$ which avoid every pattern in the set $T$. For every self-conjugate shape $\mu$,

$$
\mu_{\text {sym }}\left(T_{123}\right)=\mu_{\text {sym }}\left(T_{321}\right) \text {. }
$$

Proof. Summing Lemma 5.1 over $1 \leq i \leq \lambda_{k}$, the number of 123-avoiding symmetric full placements on a non-square self-conjugate shape equals the number of 321-avoiding such placements. Symmetry of the RSK algorithm gives $123 \sim_{\mathcal{I}} 321$, so the number of symmetric full placements on $S Q_{n}$ which avoid 123 equals the number which avoid 321. We may then apply Theorem 3.1.

As a corollary we may exchange the prefixes 123 and 321.

Corollary 5.4. The prefixes 123 and 321 may be exchanged.

\section{Proving Lemma 5.1}

The rest of this section is devoted to proving Lemma 5.1. In doing so, we symmetrize the induction used by Babson and West [BW00] in their proof of an analogous lemma for pattern avoidance by general permutations. We start with the following lemma, a consequence of the symmetry of the RSK algorithm, which provides additional base cases that are needed for our symmetrized induction. Our proof of this lemma uses the language of $n$-involutions instead of the (equivalent) language of symmetric full placements on $S Q_{n}$.

Lemma 5.5. The number of symmetric full placements on $S Q_{n}$ which avoid the pattern 123 and whose leftmost $i$ columns avoid 12 equals the number of symmetric full placements on $S Q_{n}$ which avoid 321 and whose rightmost $i$ columns avoid 21.

Proof. 123-avoiding involutions correspond to standard Young tableaux with at most 2 columns. Those which avoid 12 in their first $i$ entries are those whose first $i$ entries form a decreasing subsequence; these correspond to tableaux with at most 2 columns and whose first column contains $1, \ldots, i$.

321-avoiding involutions which avoid 21 in their last $i$ entries may be reversed to obtain 123-avoiding permutations which avoid 12 in their first $i$ entries. These correspond to pairs $(P, Q)$ of tableaux whose common shape has at most 2 columns and in which $Q$ contains $1, \ldots, i$ in its first column. Proposition 2.2 shows that the pairs of this type which correspond to the reversal of an involution are exactly those in which $P=\operatorname{evac}\left(Q^{t}\right)^{t}$.

We use the following lemma, which symmetrizes Lemma 2.2 of [BW00], to prove Lemma 5.1 and return to its proof to finish this section. 
Lemma 5.6. Let $\lambda$ be a self-conjugate shape of length $k$, with $i<\lambda_{k}$, and $1 \leq j \leq \lambda_{k}-i$. The number of symmetric full placements on $\lambda$ which avoid 321 and which avoid 21 in the $j$ columns $i+1, \ldots, i+j$ equals the number which avoid 321 everywhere and 21 in the $j$ columns $i, \ldots, i+j-1$.

Our proof of Lemma 5.1 requires the following definition.

Definition 5.7. For a non-square self-conjugate shape $\lambda$, define $\hat{\lambda}$ to be the self-conjugate shape obtained by deleting the leftmost and rightmost columns and the top and bottom rows of $\lambda$. Figure 7 shows the shape $\lambda=\left(8^{4}, 7,5^{2}, 4\right)$ and the shape $\hat{\lambda}=\left(6^{4}, 4^{2}\right)$ obtained by applying this operation. (Note that if $\lambda$ is non-square and non-empty and $\hat{\lambda}$ is empty, then $\lambda=(1)$ or $(2,1)$.)

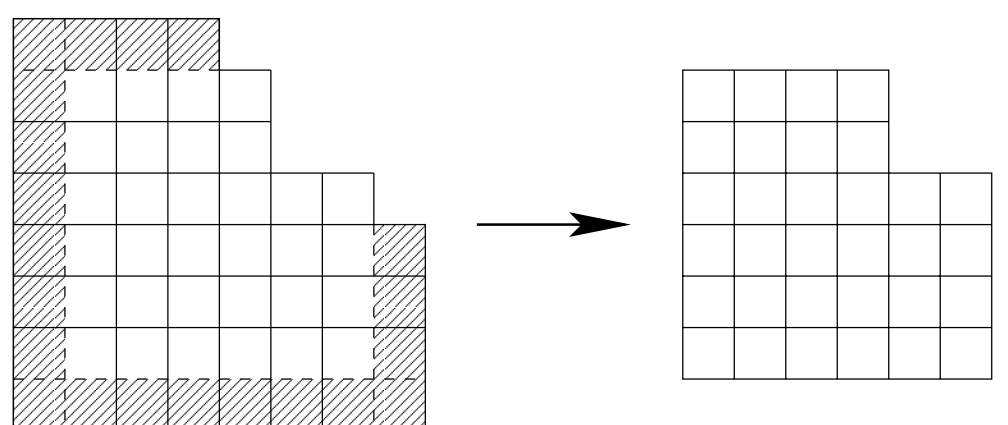

Figure 7: A non-square shape $\lambda=\left(8^{4}, 7,5^{2}, 4\right)$ and the shape $\hat{\lambda}=\left(6^{4}, 4^{2}\right)$.

We borrow notation from Babson and West to use in our symmetrized version of their proof.

Proof of Lemma 5.1. For a self-conjugate shape $\lambda$ of length $k$ and $1 \leq i \leq \lambda_{k}$, let $f_{\lambda, i}^{123}$ be the number of symmetric full placements on $\lambda$ which avoid 123 and which have a dot in $(i, k)$ (and, by symmetry, in $(k, i)$ ). Let $f_{\lambda, i}^{321}$ be the number of symmetric full placements on $\lambda$ which avoid 321 and which have a dot in $\left(\lambda_{k}+1-i, k\right)$ (and $\left(k, \lambda_{k}+1-i\right)$ ). Let $g_{\lambda, i}^{123}$ be the number of 123-avoiding symmetric full placements on $\lambda$ which also avoid 12 in the $i$ columns $1, \ldots, i$ and let $g_{\lambda, i}^{321}$ be the number of 321 -avoiding symmetric full placements on $\lambda$ which also avoid 21 in the $i$ columns $\lambda_{k}+1-i, \ldots, \lambda_{k}$. Note that the placements on $\lambda$ which avoid 12 (21) in certain columns also avoid 12 (21) in the same-labelled rows. Finally, define $g_{\lambda, 0}^{123}$ (respectively $g_{\lambda, 0}^{321}$ ) to be the number of symmetric full 123-avoiding (321-avoiding) placements on $\lambda$; we have $g_{\lambda, 0}^{123}=g_{\lambda, 1}^{123}$ and $g_{\lambda, 0}^{321}=g_{\lambda, 1}^{321}$.

Consider a symmetric full placement on a non-square shape $\lambda$ which is counted by $f_{\lambda, i}^{123}$. If $\hat{\lambda}$ is empty, $f_{\lambda, 1}^{123}=f_{\lambda, 1}^{321}=1$. If $\hat{\lambda}$ is non-empty, delete the $i^{\text {th }}$ and $k^{\text {th }}$ rows and columns of the placement on $\lambda$ to obtain a symmetric full placement on $\hat{\lambda}$ which is counted by $g_{\hat{\lambda}, i-1}^{123}$. As all such placements on $\hat{\lambda}$ can be produced this way, we have $f_{\lambda, i}^{123}=g_{\hat{\lambda}, i-1}^{123}$. Similarly, $f_{\lambda, i}^{321}$ equals the number of symmetric full placements on $\hat{\lambda}$ which avoid 321 and 
which avoid 21 in the $i-1$ columns $\lambda_{k}+1-i, \ldots, \lambda_{k}-1$. By an iterated application of Lemma 5.6, this equals $g_{\hat{\lambda}, i-1}^{321}$. If $\hat{\lambda}$ is a square, we may apply Lemma 5.5 to obtain $g_{\hat{\lambda}, i-1}^{123}=g_{\hat{\lambda}, i-1}^{321}$ and are thus finished. If $\hat{\lambda}$ is not a square, we show that $g_{\hat{\lambda}, i-1}^{123}=g_{\hat{\lambda}, i-1}^{321}$ as follows.

Let $\mu$ be a non-square self-conjugate shape of length $k$; we want to show that for $1 \leq i \leq \mu_{k}, g_{\mu, i}^{123}=g_{\mu, i}^{321}$. If $\hat{\mu}$ is empty, then there is a unique placement on $\mu$ and $g_{\mu, 1}^{123}=g_{\mu, 1}^{321}=1$ (and thus $g_{\mu, 0}^{123}=g_{\mu, 0}^{321}$ ). If $\hat{\mu}$ is non-empty, consider a placement on $\mu$ which is counted by $g_{\mu, i}^{123}$, and let $(j, k)$ be the position of the dot in the top row. If $1 \leq j \leq i$, then we must have $j=1$ since columns $1, \ldots, i$ of the placement avoid 12 . Deleting the $j^{\text {th }}\left(1^{\text {st }}\right)$ and $k^{\text {th }}$ rows and columns of $\mu$, we obtain a placement on $\hat{\mu}$ which is counted by $g_{\hat{\mu}, i-1}^{123}$ and can obtain all such placements this way. If $j>i$, then again we delete the $j^{t h}$ and $k^{\text {th }}$ rows and columns of $\mu$, thus obtaining those placements on $\hat{\mu}$ counted by $g_{\hat{\mu}, j-1}^{123}$. We thus have, for $1 \leq i \leq \mu_{k}$,

$$
g_{\mu, i}^{123}=\sum_{j=i}^{\mu_{k}} g_{\hat{\mu}, j-1}^{123} .
$$

Similarly, for $1 \leq i \leq \mu_{k}$ we also have (invoking Lemma 5.6 as above)

$$
g_{\mu, i}^{321}=\sum_{j=i}^{\mu_{k}} g_{\hat{\mu}, j-1}^{321} \text {. }
$$

We may replace any occurrences of $g_{\hat{\mu}, 0}^{123}\left(g_{\hat{\mu}, 0}^{321}\right)$ with $g_{\hat{\mu}, 1}^{123}\left(g_{\hat{\mu}, 1}^{321}\right)$. If $\mu$ is not square, we then inductively apply this argument to $\hat{\mu}$. Otherwise, we apply Lemma 5.5 to each term in these two sums.

We now complete the proof of Lemma 5.1 and Theorem 5.3 by proving Lemma 5.6. Given a 321-avoiding placement which avoids 21 in columns $i, \ldots i+j-1$ but not columns $i+1, \ldots i+j$, we construct a 321 -avoiding placement which contains 21 in columns $i, \ldots i+$ $j-1$ but not in columns $i+1, \ldots i+j$. We use a case analysis which is more extensive than that used for general permutations; the symmetrized version of the transformation used in [BW00] works in many of the cases here but requires adjustment in some cases.

Proof of Lemma 5.6. Note that the symmetry of the lemma imposes conditions on how the rows of $\lambda$ are filled; the corresponding rows must also avoid 21 (the reflection of a 21 pattern is also a 21 pattern). If $j=1$ the lemma is trivially true, so we assume that $j \geq 2$.

Fix a symmetric full 321-avoiding placement on $\lambda$ which avoids 21 in columns $i, \ldots, i+$ $j-1$ (all of which must have height $\lambda_{1}$ ). Let $l, m$, and $r$ be the number of dots in columns $i, \ldots i+j-1$ which are placed below, on, and above, respectively, the diagonal of symmetry. (Since the placement avoids 21 in these columns, within these columns any dots which lie above the diagonal must be to the right of all other dots, while any dots which lie on the diagonal must be to the right of all dots which lie below the diagonal.) 
Label columns $i, i+1, \ldots, i+j-1$ as $a_{1}, \ldots, a_{l}, b_{1}, \ldots, b_{m}, c_{1}, \ldots, c_{r}$, respectively, and denote the heights (which must increase from left to right) of the dots in these columns by $x_{1}<\cdots<x_{l}<y_{1}=b_{1}<\cdots<y_{m}=b_{m}<z_{1}<\cdots<z_{r}$.

We refer to the dots below, on, and above the diagonal and within these columns as classes $\mathbf{A}, \mathbf{B}$, and $\mathbf{C}$, respectively. Finally, let $w$ be the height of the dot in column $i+j$. Figure 8 shows dots in rows and columns $i, \ldots, i+j$ of a general placement; rows and columns $i, \ldots, i+j-1$ are marked by solid lines, while rows and columns $i+1, \ldots, i+j$ are marked by dashed lines. The three classes $\mathbf{A}, \mathbf{B}$, and $\mathbf{C}$ of dots are labelled and the other dots which must be present by the symmetry of the placement are shown. In this example, the dot in column $i+j$ has height between the heights of two dots in class $\mathbf{C}$. This placement avoids 21 in columns $i, \ldots i+j-1$ but contains 21 in columns $i+1, \ldots, i+j$; since it avoids 321 by assumption, there are various boxes (to the upper right of the figure) which the underlying Ferrers shape must not contain.

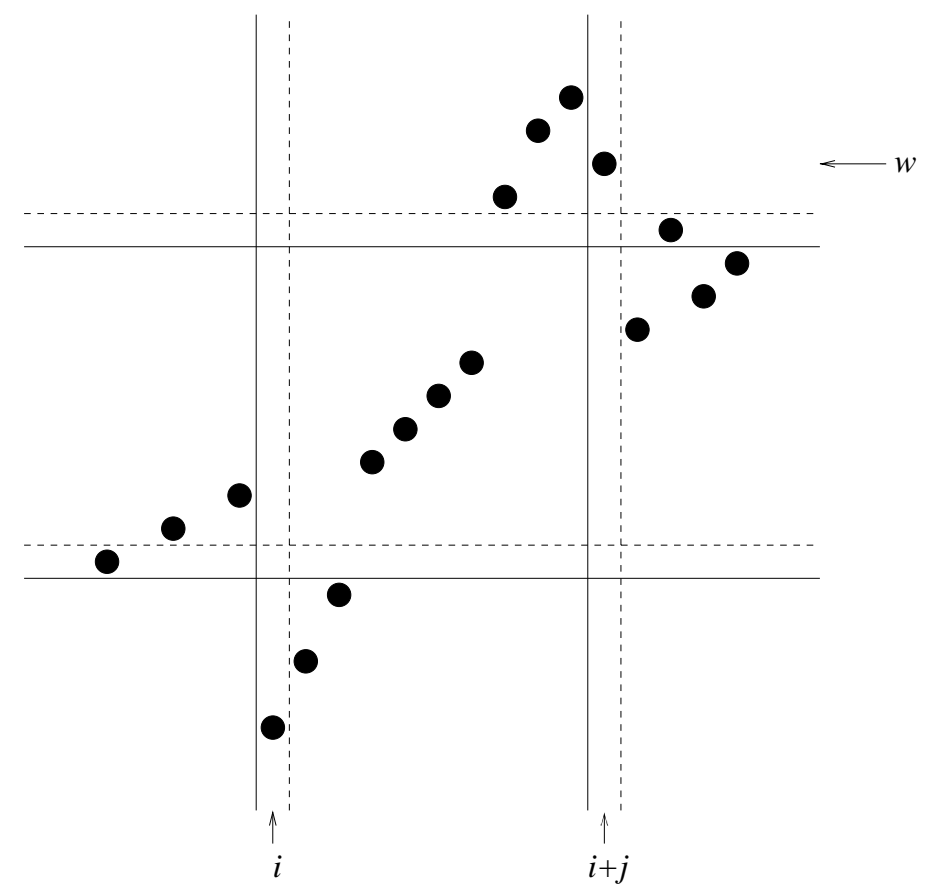

Figure 8: The setup for the proof of Lemma 5.6.

If the placement on $\lambda$ already avoids 21 in columns $i+1, \ldots, i+j$, then we leave it unchanged. If the placement contains 21 in these columns then we modify the placement so that it avoids 21 in columns $i+1, \ldots, i+j$ and contains 21 in columns $i, \ldots, i+j-1$. We define our 'basic transformation' as the symmetrization of the transformation used by Babson and West. This suffices in most cases; we modify this as needed below.

First of all, we will assume that $w \neq i+j$ and that if $w=c_{1}$ then there are no dots in class B. We may also assume that exactly the $v \geq 1$ rightmost dots in columns $i, \ldots, i+j-1$ have height greater than $w$. Our basic transformation consists of the following steps: 
(i) Move all of the dots in columns $i, \ldots, i+j-1$ except for the $v^{\text {th }}$ from the right (in column $i+j-v$, which is the dot in these columns with smallest height greater than w) 1 square to the right, keeping their heights unchanged.

(ii) Move the dot in column $i+j-v$ to column $i$ (if $v=j$ this dot does not change position).

(iii) Move the dot in column $i+j$ to column $i+j-v+1$ (if $v=1$ this dot does not change position).

(S) Symmetrize these operations, moving the dots in the corresponding rows within their columns.

Note that any dots in class $\mathbf{B}$ (on the main diagonal) are moved 1 square to the right by step (i) and then 1 square up by (S); as a result, they are still on the main diagonal in the resulting configuration. Note also that if there is a dot in columns $i, \ldots, i+j-1$ with height $i+j$ (in which case $w=c_{1}$ ), this dot will be moved by both the initial operation and the symmetrization. In other cases (with a different position of the dot in column $i+j$ or some dot classes being empty) the basic transformation has similar general effects. Figure 9 shows the placement from Figure 8 using filled circles and the transformed placement using open circles. Arrows indicate the general movement of dots, with filled arrowheads showing movements due to steps (i), (ii), and (iii) and open arrowheads the movements due to the symmetrization of these steps. The number of arrowheads indicates which step (or its symmetrization) is responsible for the movement.

After the basic transformation has been applied, the resulting placement avoids 21 in columns $i+1, \ldots, i+j$ but contains it in columns $i, \ldots, i+j-1$ since the dots which originally formed one instance of this pattern (in columns $i+j-v$ and $i+j$ ) have not changed their relative position (having moved to columns $i$ and $i+j-v+1$ ) and are still contained in the rectangular subshape of $\lambda$ formed by the leftmost $\lambda_{k}$ columns (which have height $\lambda_{1}$ ).

We proceed with a case analysis on the value of $w$, modifying the basic transformation for the case $w=c_{1}$ and determining the positions of the dots in the transformed placements. The labels of the various subcases $(e . g, \mathbf{B C})$ indicate which of the three classes of dots are nonempty. We will then show that this process is invertible.

I. $w>\max \left\{x_{l}, y_{m}, z_{r}\right\}$ In this case, columns $i+1, \ldots, i+j$ already avoid the pattern 21 and we do not modify the placement.

II. $i+j<w$ If this is not covered by case $\mathbf{I}$ then there is at least one dot in class $\mathbf{C}$ has height greater than $w$. Let $d$ be the number of dots in class $\mathbf{C}$ with heights less than $w ; w<z_{1}$ if $d=0$ and $z_{d}<w<z_{d+1}$ if $d \geq 1$. In each of the possible subcases - $\mathbf{C}, \mathbf{A C}, \mathbf{B C}$, and $\mathbf{A B C}$ - we use the basic transformation. In columns $i+1, \ldots, i+j$ of the resulting configuration, $l$ dots will lie below the main diagonal, $m$ will lie on it, and $r$ will lie above it. The dot in column $i$ will have height greater than exactly $d+1 \geq 1$ of these dots above the diagonal. 


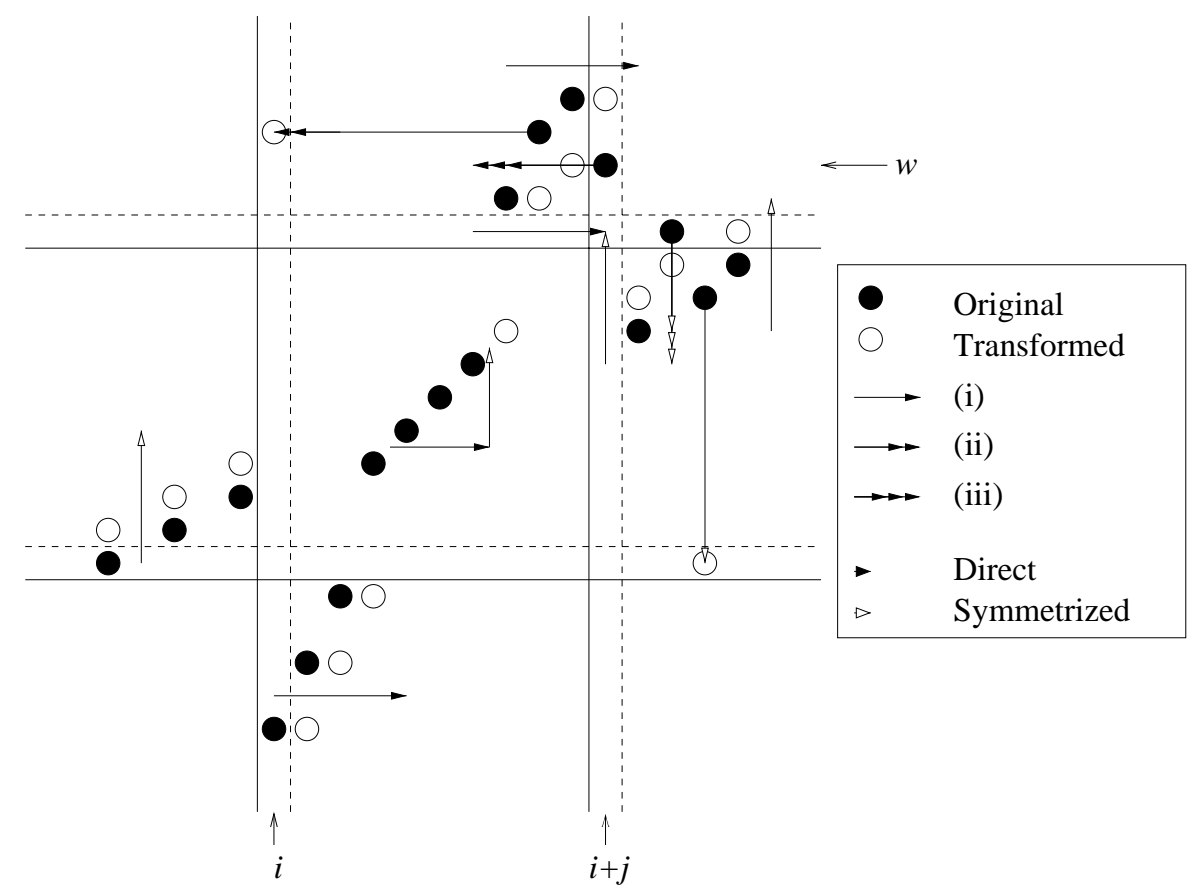

Figure 9: The basic transformation used in proving Lemma 5.6 applied to the placement in Figure 8.

III. $w=i+j$ In this case, the dot in column $i+j$ lies on the main diagonal. If the placement is not covered by case $\mathbf{I}$ then class $\mathbf{C}$ must be nonempty. In each of the possible subcases - $\mathbf{C}, \mathbf{A C}, \mathbf{B C}$, and $\mathbf{A B C}$ - we may use the basic transformation. Note that the dot initially in $(i+j, i+j)$ is moved to $\left(c_{1}+1, i+j\right)$ by step (iii) of the basic transformation and then to $\left(c_{1}+1, c_{1}+1\right)$ by the symmetrization step (S).

In columns $i+1, \ldots, i+j$ of the resulting configuration $r-1 \geq 0$ dots will lie above the main diagonal, $m+1 \geq 1$ will lie on it, and $l \geq 0$ will lie below it. The height of the dot in column $i$ will be greater than the heights of all of these dots below and on the diagonal but less than the heights of all of these dots above the diagonal.

IV. $w=c_{1}, m=0$ In this case, the point in column $i+j$ is the reflection across the main diagonal of the leftmost dot in class $\mathbf{C}$ (which is thus nonempty). Since there are no dots in class $\mathbf{B}$, we may use the basic transformation for the two possible subcases $(\mathbf{C}$ and $\mathbf{A C})$.

In columns $i+1, \ldots, i+j$ of the resulting configuration, $r-1 \geq 0$ dots lie above the main diagonal, 0 lie on it, and $l+1 \geq 1$ lie below it. The dot in column $i$ will have height greater than the heights of these dots which lie below the diagonal and less than the heights of these dots which lie above the diagonal. Note that the dots originally in $\left(i+j, c_{1}\right)$ and $\left(c_{1}, i+j\right)$ are moved to $\left(c_{1}+1, i\right)$ and $\left(i, c_{1}+1\right)$ by the combined effects of steps (i) and (S) of the basic transformation. 
V. $w=c_{1}, m>0$ In this case, the point in column $i+j$ is the reflection across the main diagonal of the leftmost dot in class $\mathbf{C}$ (which is thus nonempty). As class $\mathbf{B}$ is nonempty we need to modify the basic transformation as follows. Move the dots in columns $c_{2}, \ldots, c_{r}$ and any dots in columns $a_{1}, \ldots, a_{l}$ one box to the right and symmetrize this. (This is step (i) and its symmetrization from the basic transformation.) Combine the 2 dots at $\left(i+j, c_{1}\right)$ and $\left(c_{1}, i+j\right)$ into a single dot at $\left(c_{1}+1, c_{1}+1\right)$. Split the dot in $\left(b_{1}, b_{1}\right)$ into 2 dots at $\left(i, b_{1}+1\right)$ and $\left(b_{1}+1, i\right)$. Move any dots in columns $b_{2}, \ldots, b_{m}$ one box up and right, keeping them on the main diagonal.

In columns $i+1, \ldots, i+j$ of the resulting configuration, $r-1 \geq 0$ dots lie above the main diagonal, $m \geq 1$ lie on it, and $l+1 \geq 1$ lie below it. The dot in column $i$ has height greater than exactly $l+1$ of these dots.

VI. $w<i$ In this case, class $\mathbf{B}$ must be empty since the dots $(w, i+j),\left(y_{1}, b_{1}\right)$, and $(i+j, w)$ would form a 321 pattern. Any dots in class $\mathbf{A}$ must have height less than $w$, since if $x_{l}>w$ then the dots $(w, i+j),\left(x_{l}, a_{l}\right)$, and $(i+j, w)$ would form a 321 pattern. We use the basic transformation for the subcases - $\mathbf{C}$ and $\mathbf{A C}-$ not covered by case $\mathbf{I}$ above.

In columns $i+j, \ldots, i+j$ of the resulting configuration, $r-1 \geq 0$ dots lie above the main diagonal, 0 lie on it, and $l+1 \geq 1$ lie below it. The dot in column $i$ will have height greater than $i+j$ and less than the heights of any of these dots above the diagonal.

The basic transformation is invertible; the modified version used in case $\mathbf{V}$ is also invertible and has an image which does not meet that of the basic transformation.

We now see that the possible arrangements of 321-avoiding placements which avoid 21 in columns $i+1, \ldots, i+j$ are all in the image of one of the transformations above. Let $u$ be the height of the dot in column $i$. If $u$ is less than the heights of all the dots in columns $i+1, \ldots, i+j$ we do nothing (case I above); this covers all $u \leq i$. If $i<u \leq i+j$ then the arrangement is in the image of either case IV (if there are no dots on the diagonal in these columns) or case $\mathbf{V}$ (if there are). If $i+j<u$ and there are no dots in these columns above the diagonal with height less than $u$ then the arrangement is in the image of either case VI (if there are no dots on the diagonal in these columns) or case III (if there are). Finally, if $i+j<u$ and there is at least one dot in these columns with height less than $u$, then the arrangement is in the image of case II above.

\section{Classifying avoided patterns}

Corollary 4.3 implies many of the previously known $\sim_{\mathcal{I}}$-equivalences between symmetry classes in $\mathcal{S}_{4}$. The most notable of these is $1234 \sim_{\mathcal{I}} 2143$, originally conjectured by Guibert [Gui95] and proved more recently (using different arguments than we use) by Guibert, Pergola, and Pinzani [GPP01]. 


\section{Corollary 6.1 (Guibert, Pergola, Pinzani [GPP01]).}

$$
1234 \sim_{\mathcal{I}} 2134 \sim_{\mathcal{I}} 2143
$$

Proof. Apply Corollary 4.3 to both members of the symmetry class $\{1243,2134\}$.

Corollary 5.4 implies an affirmative answer to a conjecture of Guibert (Conjecture 5.3 of [GPP01], originally from [Gui95]) as follows.

\section{Corollary 6.2.}

$$
1234 \sim_{\mathcal{I}} 3214
$$

Corollary 6.2 completes the classification of $\mathcal{S}_{4}$ according to $\sim_{\mathcal{I}^{-}}$equivalence; we show this classification in Table 1. The leftmost column contains the symmetry classes of $\mathcal{S}_{4}$, denoted by braces $\{\cdot\}$. Double lines mark the partition of the set of symmetry classes into cardinality classes. The remaining columns give $\mathcal{I}_{n}(\tau)$ for $\tau$ in each of the cardinality classes and $5 \leq n \leq 11$. As these sequences differ from each other, there can be no other $\sim_{\mathcal{I}^{-}}$equivalences in $\mathcal{S}_{4}$.

\begin{tabular}{|l|r|r|r|r|r|r|r|}
\hline$\tau$ & $\mathcal{I}_{5}(\tau)$ & $\mathcal{I}_{6}(\tau)$ & $\mathcal{I}_{7}(\tau)$ & $\mathcal{I}_{8}(\tau)$ & $\mathcal{I}_{9}(\tau)$ & $\mathcal{I}_{10}(\tau)$ & $\mathcal{I}_{11}(\tau)$ \\
\hline \hline$\{1324\}$ & 21 & 51 & 126 & 321 & 820 & 2160 & 5654 \\
\hline \hline$\{1234\}$ & 21 & 51 & 127 & 323 & 835 & 2188 & 5798 \\
$\{1243,2134\}$ & & & & & & & \\
$\{1432,3214\}$ & & & & & & & \\
$\{2143\}$ & & & & & & & \\
$\{3412\}$ & & & & & & & \\
$\{4321\}$ & & & & & & & \\
\hline \hline$\{4231\}$ & 21 & 51 & 128 & 327 & 858 & 2272 & 6146 \\
\hline \hline$\{2431,4132$, & 24 & 62 & 154 & 396 & 992 & 2536 & 6376 \\
$3241,4213\}$ & & & & & & & \\
\hline \hline$\{1342,1423$, & 24 & 62 & 156 & 406 & 1040 & 2714 & 7012 \\
$2314,3124\}$ & & & & & & & \\
\hline \hline$\{2341,4123\}$ & 25 & 66 & 170 & 441 & 1124 & 2870 & 7273 \\
\hline \hline$\{3421,4312\}$ & 25 & 66 & 173 & 460 & 1218 & 3240 & 8602 \\
\hline \hline$\{2413,3142\}$ & 24 & 64 & 166 & 456 & 1234 & 3454 & 9600 \\
\hline
\end{tabular}

Table 1: The completed classification of $\mathcal{S}_{4}$ according to pattern avoidance by involutions. Values of $\mathcal{I}_{n}(\tau)$ for $\tau \in \mathcal{S}_{4}$ and $5 \leq n \leq 11$

In light of the results of Sections 4 and 5 , it is natural to conjecture that similar theorems hold for $12 \ldots k$ and $k \ldots 21$. 
Conjecture 6.3. For every $k$, the prefixes $12 \ldots k$ and $k \ldots 21$ may be exchanged.

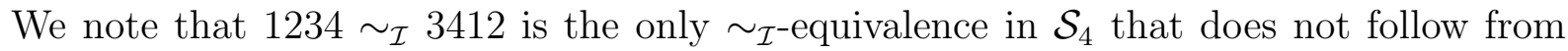
Conjecture 6.3. More generally, we also make the following conjecture.

Conjecture 6.4. Let $\mu_{\text {sym }}(\sigma)$ be the number of symmetric full placements on the shape $\mu$ which avoid the pattern $\sigma$. For every $k$ and self-conjugate shape $\mu$,

$$
\mu_{\text {sym }}(12 \ldots k)=\mu_{\text {sym }}(k \ldots 21)
$$

As noted above, Backelin, West, and Xin [BWX] have recently proved the analogue of this conjecture for pattern avoidance by general permutations.

Corollary 4.3 and 5.4 also imply (apparently new) $\sim_{\mathcal{I}^{-}}$equivalences for patterns in $\mathcal{S}_{5}$. Numerical results indicate that among the non-involutions in $\mathcal{S}_{5}$ there is only one possible $\sim_{\mathcal{I}^{-}}$equivalence, while Corollary 4.3 implies that this does indeed hold.

\section{Corollary 6.5.}

$$
12453 \sim_{\mathcal{I}} 21453
$$

Among the involutions of length 5, one cardinality class contains at most the symmetry

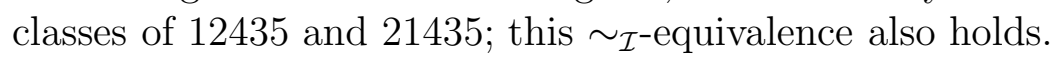

\section{Corollary 6.6.}

$$
12435 \sim_{\mathcal{I}} 21435
$$

Numerical results suggest that many symmetry classes may belong to the same cardinality class as $\{12345\}$. Corollaries 4.3 and 5.4 imply some of these possible $\sim_{\mathcal{I}^{-}}$equivalences.

\section{Corollary 6.7.}

$$
21543 \sim_{\mathcal{I}} 12543 \sim_{\mathcal{I}} 12345 \sim_{\mathcal{I}} 12354 \sim_{\mathcal{I}} 21354
$$

Recall that even without Conjecture 6.3 , we have $12 \ldots k \sim_{\mathcal{I}} k \ldots 21$ by the symmetry of the RSK algorithm.

Table 2 displays the involutions in $\mathcal{S}_{5}$ using the same format as Table 1, with the addition of single lines to separate symmetry classes with identical numerical results but which have not yet been proven to be in the same cardinality class. Table 3 shows the non-involutions in $\mathcal{S}_{5}$ in this format.

We conjecture that both of the possible equivalences consistent with Table 2 do hold.

\section{Conjecture 6.8.}

$$
12345 \sim_{\mathcal{I}} 43215
$$

\section{Conjecture 6.9.}

$$
12345 \sim_{\mathcal{I}} 45312
$$




\begin{tabular}{|l|r|r|r|r|r|r|}
\hline$\tau$ & $\mathcal{I}_{6}(\tau)$ & $\mathcal{I}_{7}(\tau)$ & $\mathcal{I}_{8}(\tau)$ & $\mathcal{I}_{9}(\tau)$ & $\mathcal{I}_{10}(\tau)$ & $\mathcal{I}_{11}(\tau)$ \\
\hline \hline$\{35142,42513\}$ & 70 & 195 & 582 & 1725 & 5355 & 16510 \\
\hline \hline$\{14325\}$ & 70 & 196 & 587 & 1757 & 5504 & 17220 \\
\hline \hline$\{12435,13245\}$ & 70 & 196 & 587 & 1759 & 5512 & 17290 \\
$\{13254,21435\}$ & & & & & & \\
\hline \hline$\{12345\}$ & 70 & 196 & 588 & 1764 & 5544 & 17424 \\
$\{54321\}$ & & & & & & \\
$\{12354,21345\}$ & & & & & & \\
$\{12543,32145\}$ & & & & & & \\
$\{21354\}$ & & & & & & \\
$\{21543,32154\}$ & & & & & & \\
\hline$\{15432,43215\}$ & 70 & 196 & 588 & 1764 & 5544 & 17424 \\
\hline$\{45312\}$ & 70 & 196 & 588 & 1764 & 5544 & 17424 \\
\hline \hline$\{52431,53241\}$ & 70 & 196 & 588 & 1764 & 5544 & 17426 \\
\hline \hline$\{52341\}$ & 70 & 196 & 589 & 1773 & 5604 & 17768 \\
\hline \hline$\{14523,34125\}$ & 70 & 197 & 592 & 1791 & 5644 & 17900 \\
\hline \hline$\{15342,42315\}$ & 70 & 197 & 593 & 1797 & 5685 & 18101 \\
\hline
\end{tabular}

Table 2: Values of $\mathcal{I}_{n}(\tau)$ for involutions $\tau \in \mathcal{S}_{5}$ and $6 \leq n \leq 11$.

The first of these follows from Conjecture 6.3 , while the second is the only $\sim_{\mathcal{I}}$-equivalence which is consistent with the numerical results presented here but which would not follow from Conjecture 6.3.

Table 4 uses a somewhat different format to present just those involutions $\tau \in \mathcal{S}_{6}$ for which $\mathcal{I}_{n}(\tau)=\mathcal{I}_{n}(123456)$ for $7 \leq n \leq 11$. Single lines now separate symmetry classes

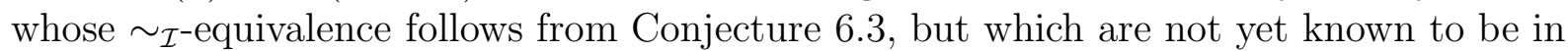
the same cardinality class, while double lines separate the other symmetry classes whose numerical results agree with those of 123456 . Symmetry classes which are not separated by any lines are known to be in the same cardinality class.

A natural question is whether there are any $\sim_{\mathcal{I}^{-}}$equivalences between 123456 and other patterns which do not follow from Conjecture 6.3.

Question. Does either $123456 \sim_{\mathcal{I}} 456123$ or $123456 \sim_{\mathcal{I}} 564312$ hold?

These resemble the known $\sim_{\mathcal{I}^{-}}$-equivalence $1234 \sim_{\mathcal{I}} 3412$ and the conjectured $\sim_{\mathcal{I}}$-equivalence $12345 \sim_{\mathcal{I}} 45312$, the only possible $\sim_{\mathcal{I}}$-equivalences in $\mathcal{S}_{4}$ and $\mathcal{S}_{5}$ which do not follow from Conjecture 6.3. It is natural to ask whether these follow from some other general theorem. Such a general result could not be stated in terms of prefix exchanging as $12345 \chi_{\mathcal{I}} 34125$ and $123456 \chi_{\mathcal{I}} 453126$ (although not shown in Table $4, \mathcal{I}_{8}(453126)=$ $\left.716>715=\mathcal{I}_{8}(123456)\right)$.

Question. Is there a general theorem which implies $1234 \sim_{\mathcal{I}} 3412,12345 \sim_{\mathcal{I}} 45312$, and one or both of $123456 \sim_{\mathcal{I}} 456123$ and $123456 \sim_{\mathcal{I}} 564312 ?$ 


\begin{tabular}{|l|r|r|r|r|r|c|}
\hline$\tau$ & $\mathcal{I}_{6}(\tau)$ & $\mathcal{I}_{7}(\tau)$ & $\mathcal{I}_{8}(\tau)$ & $\mathcal{I}_{9}(\tau)$ & $\mathcal{I}_{10}(\tau)$ & $\mathcal{I}_{11}(\tau)$ \\
\hline \hline$\{13542,42135,15243,32415\}$ & 74 & 214 & 644 & 1945 & 6004 & 18526 \\
\hline \hline$\{13425,14235\}$ & 74 & 214 & 647 & 1959 & 6107 & 18952 \\
\hline \hline$\{14352,41325,15324,24315\}$ & 74 & 215 & 649 & 1975 & 6126 & 19057 \\
\hline \hline$\{25431,53214,43251,51432\}$ & 74 & 216 & 654 & 2002 & 6223 & 19425 \\
\hline \hline$\{45231,53412\}$ & 74 & 216 & 656 & 2020 & 6342 & 20072 \\
\hline \hline$\{12453,31245,12534,23145\}$ & 74 & 216 & 656 & 2022 & 6362 & 20212 \\
$\{21453,31254,21534,23154\}$ & & & & & & \\
\hline \hline$\{25143,32514,31542,42153\}$ & 74 & 216 & 658 & 2033 & 6434 & 20538 \\
\hline \hline$\{13452,41235,15234,23415\}$ & 75 & 220 & 674 & 2067 & 6463 & 20150 \\
\hline \hline$\{13524,24135,14253,31425\}$ & 74 & 217 & 664 & 2068 & 6598 & 21269 \\
\hline \hline$\{14532,43125,15423,34215\}$ & 75 & 220 & 677 & 2090 & 6609 & 20880 \\
\hline \hline$\{32541,52143\}$ & 75 & 221 & 679 & 2096 & 6577 & 20630 \\
\hline \hline$\{25341,52314,42351,51342\}$ & 75 & 221 & 680 & 2103 & 6617 & 20808 \\
\hline \hline$\{35241,52413,42531,53142\}$ & 75 & 220 & 680 & 2111 & 6745 & 21567 \\
\hline \hline$\{24513,35124,34152,41523\}$ & 75 & 221 & 682 & 2122 & 6752 & 21569 \\
\hline \hline$\{53421,54231\}$ & 74 & 218 & 672 & 2126 & 6908 & 22877 \\
\hline \hline$\{24351,51324\}$ & 75 & 222 & 687 & 2136 & 6735 & 21093 \\
\hline \hline$\{23541,52134,32451,51243\}$ & 75 & 222 & 687 & 2137 & 6737 & 21132 \\
\hline \hline$\{45321,54312\}$ & 75 & 222 & 688 & 2156 & 6892 & 22128 \\
\hline \hline$\{23514,25134,31452,41253\}$ & 75 & 222 & 688 & 2159 & 6923 & 22358 \\
\hline \hline$\{35412,45213,43512,45132\}$ & 75 & 222 & 689 & 2168 & 6981 & 22676 \\
\hline \hline$\{25413,35214,41532,43152\}$ & 75 & 222 & 690 & 2172 & 7004 & 22731 \\
\hline \hline$\{23451,51234\}$ & 75 & 223 & 694 & 2183 & 6958 & 22127 \\
\hline \hline$\{35421,54213,43521,54132\}$ & 75 & 223 & 696 & 2209 & 7177 & 23533 \\
\hline \hline$\{24153,31524\}$ & 75 & 224 & 701 & 2240 & 7315 & 24190 \\
\hline \hline$\{24531,53124,34251,51423\}$ & 76 & 227 & 715 & 2257 & 7269 & 23254 \\
\hline \hline$\{34512,45123\}$ & 75 & 224 & 705 & 2273 & 7538 & 25418 \\
\hline \hline$\{25314,41352\}$ & 76 & 228 & 722 & 2302 & 7514 & 24530 \\
\hline \hline$\{34521,54123\}$ & 76 & 230 & 732 & 2364 & 7764 & 25596 \\
\hline
\end{tabular}

Table 3: Values of $I_{n}(\tau)$ for non-involutions $\tau \in \mathcal{S}_{5}$ and $6 \leq n \leq 11$. 


\begin{tabular}{|l|r|r|r|r|r|}
\hline$\tau$ & $\mathcal{I}_{7}(\tau)$ & $\mathcal{I}_{8}(\tau)$ & $\mathcal{I}_{9}(\tau)$ & $\mathcal{I}_{10}(\tau)$ & $\mathcal{I}_{11}(\tau)$ \\
\hline \hline$\{123456\}$ & 225 & 715 & 2347 & 7990 & 27908 \\
$\{123465,213456\}$ & & & & & \\
$\{123654,321456\}$ & & & & & \\
$\{213465\}$ & & & & & \\
$\{213654,321465\}$ & & & & & \\
$\{321654\}$ & & & & & \\
$\{654321\}$ & & & & & \\
$\{126543,432156\}$ & 225 & 715 & 2347 & 7990 & 27908 \\
$\{216543,432165\}$ & & & & & \\
\hline$\{165432,543216\}$ & 225 & 715 & 2347 & 7990 & 27908 \\
\hline \hline$\{456123\}$ & 225 & 715 & 2347 & 7990 & 27908 \\
\hline \hline$\{564312\}$ & 225 & 715 & 2347 & 7990 & 27908 \\
\hline
\end{tabular}

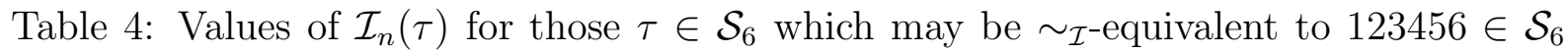
and $7 \leq n \leq 11$.

Stankova and West [SW02] proved that 231 and 312 are shape-Wilf-equivalent. However, we cannot have $\mu_{\text {sym }}(231)=\mu_{\text {sym }}(312)$ for every self-conjugate shape $\mu$ since $\mathcal{I}_{10}(231564)=8990<8991=\mathcal{I}_{10}(312564)$. Stankova and West also noted a 'sporadic' case of Wilf-equivalence (between the $\sim_{\mathcal{S}}$-symmetry classes of 1342 and 3142 ) which does not follow from any known instance of shape-Wilf-equivalence. It is interesting to see that this Wilf-equivalence breaks when we pass to $\sim_{\mathcal{I}}$-cardinality classes; neither of the two $\sim_{\mathcal{I}}$-symmetry classes contained in the $\sim_{\mathcal{S}}$-symmetry class of 1342 are $\sim_{\mathcal{I}}$-equivalent to the $\sim_{\mathcal{I}}$-symmetry class containing 3142 (which in also the $\sim_{\mathcal{S}}$-symmetry class of 3142 ).

There are also asymptotic questions which can be asked for pattern avoidance by involutions. It seems reasonable to ask, as Stankova and West have conjectured for the general permutation case, whether the cardinality classes can be ordered asymptotically. For relatively small values of $n$, it is reasonable to expect that if $\sigma$ is an involution and $\pi$ is not then $\mathcal{I}_{n}(\sigma)<\mathcal{I}_{n}(\pi)$. However, comparing the growth rates of, e.g., $\mathcal{I}_{n}(15342)$ and $\mathcal{I}_{n}(13542)$, suggests that it may be too optimistic to expect this to hold for all $n$. Finally, it would be of interest to find the asymptotic growth of $\mathcal{I}_{n}(\tau)$ for those $\tau \chi_{\mathcal{I}} 12 \ldots k$.

\section{Acknowledgements}

We are grateful to Herb Wilf for helpful discussions about this work, the organizers of and participants in the Permutation Patterns 2003 conference for stimulating interactions about current pattern work, and Andre Scedrov for arranging research support. We also thank the anonymous referee for a number of useful suggestions. 


\section{References}

[BW00] Eric Babson and Julian West, The permutations $123 p_{4} \cdots p_{m}$ and $321 p_{4} \cdots p_{m}$ are Wilf-equivalent, Graphs Combin. 16 (2000), no. 4, 373-380.

[BWX] Jörgen Backelin, Julian West, and Guoce Xin, Wilf-equivalence for singleton classes, Preprint.

[GB89] Dominique Gouyou-Beauchamps, Standard Young tableaux of height 4 and 5, European J. Combin. 10 (1989), no. 1, 69-82.

[Ges90] Ira M. Gessel, Symmetric functions and P-recursiveness, J. Combin. Theory Ser. A 53 (1990), no. 2, 257-285.

[GM02] O. Guibert and T. Mansour, Restricted 132-involutions, Sém. Lothar. Combin. (2002), Article \#B48a, 23pp. (electronic).

[GPP01] O. Guibert, E. Pergola, and R. Pinzani, Vexillary Involutions are Enumerated by Motzkin Numbers, Ann. Comb. 5 (2001), no. 2, 153-174.

[Gui95] O. Guibert, Combinatoire des permutations à motifs exclus en liaison avec mots, cartes planaires et tableaux de young, Ph.D. thesis, University Bordeaux 1, 1995.

[Reg81] Amitai Regev, Asymptotic values for degrees associated with strips of Young diagrams, Adv. in Math. 41 (1981), no. 2, 115-136.

[Rei03] Astrid Reifegerste, A generalization of Simion-Schmidt's bijection for restricted permutations, Electron. J. Combin. 9 (2003), no. 2, Paper \#R14, 9 pp. (electronic).

[Sch63] M. P. Schützenberger, Quelques remarques sur une construction de Schensted, Math. Scand. 12 (1963), 117-128.

[SS85] Rodica Simion and Frank W. Schmidt, Restricted permutations, European J. Combin. 6 (1985), no. 4, 383-406.

[Sta99] Richard P. Stanley, Enumerative combinatorics. Vol. 2, Cambridge University Press, Cambridge, 1999.

[SW02] Zvezdelina Stankova and Julian West, A new class of Wilf-equivalent permutations, J. Algebraic Combin. 15 (2002), no. 3, 271-290.

[Wes90] Julian West, Permutations with forbidden subsequences; and, Stack-sortable permutations, Ph.D. thesis, M.I.T., 1990. 